BRUGGER E.A. 1982: Regionale Strukturpolitik in Entwicklungsländern. Diessenhofen.

BRUGGER E.A. 1981: Regionalforschung als Chance der Wirtschafts- und Spezialgeographie. In: Geogr. Helvetica, 4/81, S. 167-175

EISEL U. 1981: Zum Paradigmawechsel in der Geographie. In: Geogr. Helvetica 4/81, S.176-189.

NYDEGGER A./OBERHÄNSLI H./HARRINGER R. 1983: Investitionen und Innovationen in kleineren Industriebetrieben. Diessenhofen.
MAILLAT D. (Ed.) 1983: Technology - a key factor for regional development. St. Saphorin.

RAPOPORT A. 1982: Gegenüberstellung von zwei Ansätzen zur allgemeinen Systemtheorie. Vortragsmanuskript. Wien.

STIENS G. 1980: Zur Wiederkunft des Regionalismus in den Wissenschaften. Informationen zur Raumentwicklung 5/80, S. 315-333.

STUCKEY B. 1980: Economic Progress: Illusion and reality. In: Ethnologie im Dialog, hrsg. von G.BAER/P. CENTLIVRES. Fribourg. S. 135-180.

\title{
2.3 Marktlücken als Chancen für Geographieabsolventen?
}

\begin{abstract}
Wenn wir von den Schwierigkeiten der Stellensuche hören, die ausstudierte Geographen haben, wenn sie ins Berufsleben eintreten wollen - um in ihrem Fachgebiet zu arbeiten, wohlgemerkt -, müssen wir uns die Frage stellen, ob ein Bedarf an Geographen überhaupt vorhanden ist. Sicher war die Situation vor ein paar Jahren schlimmer; heute mit steigendem Umweltbewußtsein erhält auch die Stellung des Geographen mehr Bedeutung. Allerdings ist auch er nicht darum herumgekommen, sich zu spezialisieren. Die prekäre Stellensituation hat nun im Geographischen Institut der Universität Bern dazu geführt, da $\beta$ in verschiedenen Abteilungen (v. a. der physikalischen Richtung) Initiativen ergriffen wurden, um in eigenwirtschaftlicher Verantwortung in den speziellen Gebieten selber Arbeitsplätze zu schaffen. METEOTEST darf sich das Verdienst gutschreiben, bei den ersten gewesen zu sein, die solche Pläne konkret umgesetzt haben. Später haben Geomorphologen nachgezogen; es wird eine kleine Firma betrieben, die sich auf Luftbildaufnahmen mit einer $6 \times 6$-Kamera spezialisiert hat; und neuerdings haben auch Hydrologen damit begonnen, auf privater Basis zu arbeiten.

Es scheint also, daß Marktlücken erstens existieren und daß sie zweitens von Geographen als Chancen aufgefaßt werden, in ihrem Beruf tätig zu sein.
\end{abstract}

\subsubsection{Als Beispiel: METEOTEST}

Der Wunschtraum eines jeden Studenten ist es, das an der Universität vermittelte theoretische Wissen auch praktisch anwenden zu können.
So war auch in der Klimaabteilung des geographischen Instituts der Universität Bern (GIUB) schon lange die Idee aufgekommen, ein auf privatwirtschaftlicher Basis geführtes «Büro für angewandte Klimatologie» zu gründen. Man rechnete sich einige Chancen aus, auf dem Gebiet der angewandten Klimatologie einen Marktanteil «ergattern» zu können. Dieser Optimismus beruhte nicht zuletzt auf der Tatsache, da $\beta$ häufig von privater Seite oder von amtlichen Stellen an die Klimatologen des GIUB kleinere oder auch größere bezahlte Aufträge herangetragen wurden. Meist scheiterte deren Ausführung jedoch daran, da $\beta$ sie entweder relativ dringend waren und kurzfristig kein Personal zur Verfügung stand oder daß sie zu weit abseits der allgemeinen Forschungsrichtung standen und deshalb von vornherein abgelehnt wurden.

Zu Beginn des Jahres 1981 begannen sich deshalb einige Angehörige der Klimaabteilung des GIUB daranzumachen, die Gründung eines solchen privaten Büros voranzutreiben. Man war sich einig darüber, eine von der Universität unabhängige Firma aufbauen zu wollen. Sie sollte auch eine rechtliche Form erhalten; wir entschlossen uns, eine Genossenschaft mit Namen METEOTEST zu gründen, die unter diesem Namen ein Büro für angewandte Klimatologie betreiben sollte. In diesem Sinn schufen wir Statuten

Martin Baumgartner, Jürg Engel

Meteotest, Büro für angewandte Klimatologie, Hallerstrasse 50 , 3012 Bern 
und meldeten die Genossenschaft beim Handelsregister an. Der Betrieb des Büros obliegt einer 3-köpfigen Geschäftsleitung, die in erster Linie dem Verwaltungsrat (ebenfalls 3 Mitglieder) verantwortlich ist. Geschäftsleitung und Verwaltungsrat werden von der Generalversammlung gewählt. Alle Mitglieder der Genossenschaft METEOTEST sind ausgebildete Geographen, die sich auf den verschiedensten Gebieten der Klimatologie spezialisiert haben.

Mit Beginn des Betriebs am 8. September 1981 tauchten auch die ersten Probleme auf. Wir kamen ja direkt von der Universität und waren in allen geschäftlichen Belangen völlig unerfahren. Auch unsere finanziellen Mittel - die Anteilscheineinlagen der Genossenschafter - waren sehr knapp. Sie wurden nur dazu verwendet, die Infrastruktur und den Betrieb des Büros zu bestreiten. Glücklicherweise konnten wir einen günstigen Büroraum genügender $\mathrm{Größe}$ in guter Lage anmieten. Am Anfang stellten die Genossenschafter das Büromobiliar zur Verfügung, mit der Zeit wurde es dann aber mit genossenschaftseigenem ersetzt. Die administrativen Arbeiten erledigte hauptsächlich die Geschäftsleitung in unentgeltlicher Fronarbeit. Ein weiterer glücklicher Umstand war es, daß wir gleich zu Beginn einen mittleren Auftrag in Arbeit bekamen, der einerseits für 2 bis 3 Leute stundenweise Verdienst ergab, andererseits die Kapitalerhaltung ermöglichte.

Der Einstieg in den «Markt» erwies sich aber als nicht ganz so einfach. Obwohl aus der Sicht des Klimatologen/Geographen ein genügend großes Tätigkeitsfeld vorhanden ist, weiß die Öffentlichkeit zu wenig über die Ausbildung und die Möglichkeiten der Klimatologen. Dementsprechend werden die spezifischen Aufgaben auch häufig an Architekten, Architekten-Planer und Ingenieure verschiedenster Provenienz vergeben. Unsere große Konkurrenz sind damit die etablierten Architektur- und Ingenieurbüros, die sich - vielleicht zwangsläufig - auch auf Fragen der Klimatologie spezialisiert haben. Die Problematik, zu einem Marktanteil zu kommen, liegt nun also darin, das Bild des Klimatologen gegen außen in ein richtiges Licht zu rücken und die Öffentlichkeit bzw. die potentiellen Auftraggeber über das Profil des Klimatologen zu informieren. Ein weiterer wichtiger Punkt ist aber sicher auch der, daß bekanntgemacht werden muß, da $\beta$ jetzt ein privates Spezialbüro existiert, das sich mit spezifischen klimatischen Fragestellungen beschäftigt.

Wir sehen unseren Tätigkeitsbereich vor allem in der Anwendung bekannter Methoden der Klimatologie, eben angewandte Klimatologie. Von der Thematik her stehen uns viele Möglichkeiten offen: Alternativenergien (Strahlungsangebot, Windverhältnisse), Umweltschutz (Schadstoffausbreitung, Temperaturschichtung, Durchlüftung usw.), Lokalklima (Landwirtschaft, Wohnsiedlungsbau, allgemeine Planungsfragen) und mehr. Wir haben v. a. auch die Möglichkeit, selber klimatische Messungen durchzuführen (siehe nächstes Kapitel). Aber auch weiterreichende Aufträge, z.B. Arbeiten im Ausland, interdisziplinäre Zusammenarbeit, Forschungsfragen, liegen im Bereich unseres Tätigkeitsfeldes.

\subsubsection{Typische Arbeitsbereiche für METEOTEST}

\subsubsection{Einleitung}

Im Verlaufe der vergangenen Geschäftsjahre war unser Büro schwerpunktmäßig auf drei verschiedenen Gebieten tätig:

- redaktionelle Arbeiten

Erarbeiten und Überarbeiten von regionalen und fachspezifischen Studien.

- Sonnenenergienutzung bzw. -angebot

Berechnen der Sonnenscheindauer (effektiv und absolut) und der Direktstrahlung. Wir verwenden dazu zur Erfassung des Horizonts eine Fischaugenkamera und verfügen über die entsprechende Software, welche die gewünschten Resultate liefert.

- klimatologische Messungen

Planung, Durchführung, Auswertung und Interpretation von Messungen verschiedener Klimaelemente. Es steht uns ein großer Gerätepark zur Verfügung.

Im folgenden wollen wir als Beispiel die meßtechnische Seite einer von uns durchgeführten Untersuchung im Hinblick auf lufthygienische Fragestellungen kurz beleuchten.

\subsubsection{Durchführung einer meteorologischen Meß- kampagne in einer Kleinregion}

In einer bestimmten kleineren Region sollen alle zur Beurteilung der Durchlüftungsverhältnisse notwendigen Klimaelemente erfaßt und beurteilt werden. Unter «Durchlüftung» wird die Ersetzung der mit lokalen Eigenschaften behafteten Luft durch solche, die auf dem Wege der Großzirkulation herangeführt wird, verstanden. Es führen vor allem Wetterlagen mit schwachen Winden und Temperaturinversionen (u. a. Hoch- und Flachdrucklagen im Herbst und Winter) zu einem gehemmten Luftaustausch (horizontal und vertikal). Bei solchen Lagen besteht eine potentielle Gefahr der Luftfremdstoffanreicherung $\left(\mathrm{CO}, \mathrm{SO}_{2}\right.$, $\mathrm{NO}_{\mathrm{x}}, \mathrm{CH}, \mathrm{O}_{3}$, Staub, Schwermineralien usw.), insbesondere in Tälern und Becken, vor allem wenn sich die Inversion während Tagen nicht aufzulösen vermag.

Um diese Durchlüftungsverhältnisse in der Untersuchungsregion abschätzen zu können, muß eine großangelegte Meßkampagne durchgeführt werden.

a) Stationäres Meßnetz:

Während einer längeren Periode (für die erwähnte Problemstellung am besten während einiger Wintermonate) werden an entscheidenden Lokalitäten 
mehrere Windmesser und Temperatur-Feuchtemesser mit kontinuierlicher Registrierung betrieben. Die Kontrolle dieser Geräte muß dabei wöchentlich erfolgen (Photo 1).

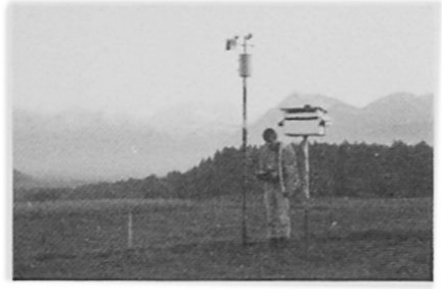

Photo 1 Kontinuierliche Messung und Aufzeichnung von Temperatur, Feuchtigkeit, Windrichtung und Windstärke

b) 24-Stunden-Meßkampagne:

Während 24 Stunden (im Winter, zur Zeit einer stabilen Hochdruckwetterlage) werden zusätzlich zum stationären Meßnetz noch folgende Meßprogramme durchgeführt (Photo 2):

- Meßfahrten mit einem speziell ausgerüsteten Meßfahrzeug entlang einer genau definierten $\mathrm{Me} \beta$ strecke: Automatische Erfassung von Temperatur, Feuchte und Luftdruck (bodennahe Horizontalprofile).

- An einem bis zwei Standorten werden Ballonsondagen ca. alle Stunden durchgeführt: Erfassung des vertikalen Temperaturprofils zur Bestimmung der Inversionsmächtigkeit.

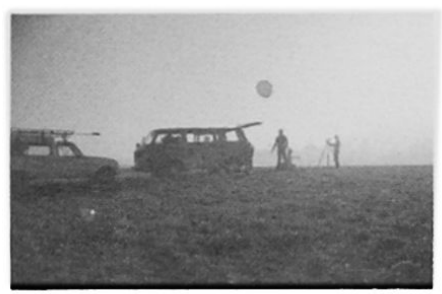

Photo 2 Einsatz des Ballonsondiersystems zur Erfassung des vertikalen Temperaturverlaufes

- Tagsüber werden aus einem Kleinflugzeug die Bodentemperaturen mittels eines Radiometers (Infrarotmeßgerät) erfaßt.

- An kritischen Stellen können Rauchversuche zur Bestimmung des bodennahen Kaltluftabflusses durchgeführt werden (Photos 3-5).

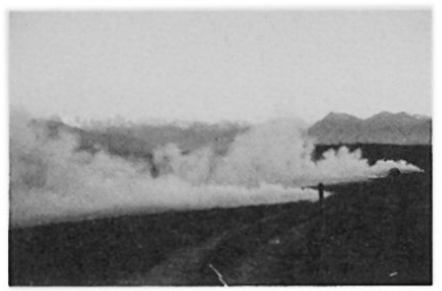

Photo 3 Durch den Rauch wird das bodennahe Abströmen der Kaltluft verdeutlicht

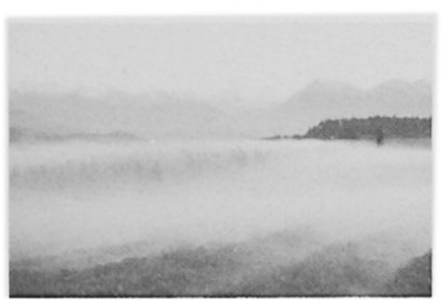

Photo 4 Anhand des Maisfeldes wird die geringe Mächtigkeit der sehr kalten bodennahen Luft ersichtlich

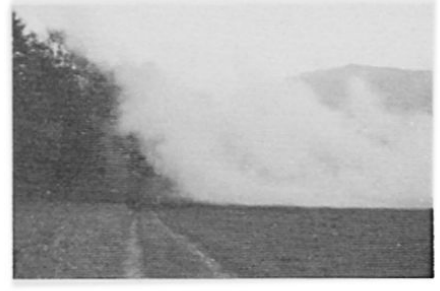

Photo 5 Kaltluftstau bedingt durch einen kleinen Wald

c) Auswertung der Daten, Probleme:

Die Auswertung aller Meßdaten erfordert größte Sorgfalt. Stehen schlussendlich alle Daten auf einem geeigneten EDV-Datenträger zur Verfügung, können die gewünschten Analysen durchgeführt werden. Das Ziel einer Untersuchung der Durchlüftungsverhältnisse ist eine "Durchlüftungskarte», welche die durchlüftungsgefährdeten Gebiete (schlechter Luftaustausch!) der Untersuchungsregion aufzeigt.

Die Projektierung, Durchführung und Auswertung einer solchen Meßkampagne bedarf eines relativ großen Zeitaufwandes. Zudem muß immer wieder mit einem Meßunterbruch, bedingt durch technische Probleme an den Meßgeräten oder aber z.T. leider auch durch mutwillige Beschädigung der Geräte durch Drittpersonen, gerechnet werden.

\subsubsection{Ausblick}

Wir haben unser Büro bisher nur mit Teilzeitanstellungen und einem großen Input an Fronarbeiten geführt. Eines unserer Ziele ist es deshalb, feste Stellen zu schaffen. Dazu aber benötigen wir ein größeres Auftragsvolumen, als das bisher der Fall gewesen ist, und eine kontinuierliche Abfolge von Aufträgen. Dies zu erreichen bedingt vermutlich eine Diversifizierung unseres Angebots. Darunter verstehen wir jedoch nicht unbedingt eine Ausweitung in andere Fachgebiete als die Klimatologie. (Von unserer geographischen Ausbildung her wäre es relativ leicht denkbar, in Bereiche der Hydrologie, Geomorphologie, Raumplanung usw. einzusteigen.) Vielmehr sehen wir Chancen auf dem Gebiet der Alternativenergie, insbesondere der Nutzung der Sonneneinstrahlung, bzw. der Abklärung des potentiellen Strahlungsangebots (Sonnenscheindauer, Direktstrahlung) eines Standorts. Auch im Zusammenhang mit Umweltschutzfragen (Stichwort: Umweltverträglichkeitsprüfung) nehmen wir an, daß der Bedarf an klimatologischen Abklärungen steigen wird. Weitere entferntere Möglichkeiten liegen in Richtung EDV-Dienstleistungenloder dem Ausbau der redaktionellen Bearbeitung von Fachberichten oder -publikationen. 